Z Rheumatol 2014 · 73:696-697

DOI 10.1007/s00393-014-1454-4

Online publiziert: 26. September 2014

c) Springer-Verlag Berlin Heidelberg 2014

R. Alten ${ }^{1} \cdot$ M. Schneider ${ }^{2}$

${ }^{1}$ Schlosspark-Klinik, Akademisches Lehrkrankenhaus der Charité - Universitätsmedizin Berlin

${ }^{2}$ Klinik für Endokrinologie, Diabetologie u. Rheumatologie, Universitätsklinikum Düsseldorf

\title{
Der Patient im Mittelpunkt
}

\section{Patient-reported outcome}

Die Weltgesundheitsorganisation hat erklärt, dass die Einbeziehung von Patienten in ihre eigene Gesundheitsversorgung nicht nur erstrebenswert ist, sondern eine soziale, ökonomische und technische Notwendigkeit. Patienten möchten mitentscheiden, insbesondere wenn Behandlungsalternativen zur Verfügung stehen also eigentlich immer. Sie tragen ohnehin die letztendliche Verantwortung für viele Entscheidungen, die im Zusammenhang mit ihrer Gesundheit getroffen werden: Sie entscheiden, wann sie einen Arzt konsultieren, ob sie die vorgeschlagenen Empfehlungen annehmen und letztlich, ob sie verordnete Medikamente einnehmen oder alternative Behandlungsstrategien suchen. Damit sie mit diesen Entscheidung nicht allein stehen oder sie ohne ihren Arzt treffen, müssen die Behandler die Erkrankten immer wieder aktiv in den Prozess mit einbinden und auch jede Entscheidung anerkennen.

Der Anerkennung von Patienten als Experten für ihren eigenen Körper Kompetenz zu übernehmen trägt die zunehmende Bedeutung von „patient-reported outcomes“ (PRO) Rechnung. Denn meist bieten uns die Betroffenen ja auch die Zielkriterien für eine erfolgreiche Therapie. Nur bisher haben wir Ärzte diese Informationen zuerst in scheinbar objektiviere Messinstrumente übersetzt, bevor sie verwertet haben. Das ändert sich jetzt zunehmend.

In den letzten Jahren sind PRO in die Empfehlungen verschiedener Institutionen aufgenommen worden. Im Februar 2006 hatte die „U.S. Food and Drug Administration“ (FDA) erste Empfehlungen zur Implementierung und Beurteilung von PRO bei Arzneimittel- und Medizinproduktzulassungen gegeben.

Anfang diesen Jahres wurde im Rahmen des Qualifikationsprozesses der FDA zur Entwicklung von validierten PROs als erstes das Instrument „Exacerbations of Chronic Pulmonary Disease Tool“ (EXACT)für die chronisch-obstruktive Lungenerkrankung (COPD) vorgestellt.

In Deutschland ist die Bedeutung der PRO aus den Vorgaben des Sozialgesetzbuchs (SGB V $\$ 35$ b Abs. 1) ersichtlich. Das Institut für Qualität und Wirtschaftlichkeit im Gesundheitswesen (IQWIG) hat im aktuellen Methodenpapier 4.1 (2013) erneut darauf hingewiesen, dass neben der gesundheitsbezogenen Lebensqualität auch andere Nutzendimensionen mittels PRO erfasst werden sollen.

Die wachsende Bedeutung von PRO wird in den Beiträgen dieses Themenhefts für verschiedene Indikationen und aus der Sicht der Patientenorganisation gewürdigt.

Der Einsatz von PRO bietet sich für zahlreiche Anwendungsgebiete beim systemischen Lupus erythematodes (SLE) an, wozu neben den Zulassungsstudien, epidemiologischen Studien und v. a. auch die Patientenversorgung im klinischen, ambulanten Alltag gehören, wie G. Che$b a b$ et al. in ihrem Beitrag „Möglichkeiten und Grenzen patientenberichteter Endpunkte am Beispiel des systemischen Lupus erythematodes und der LuLa-Studie" ausführen.

Neben der Erhebung von Schmerz, Fatigue, Depression, „health-related quality of life“ (HRQoL) und "quality of life" (QoL) können z. B. auch Krankheitsaktivität und -schaden abgebildet werden.
Die Empfehlungen der „European League Against Rheumatism“ (EULAR) zum Monitoring bei SLE-Patienten schlagen ein regelmäßiges Messen von Aktivität und Lebensqualität bei jeder Visite sowie die jährliche Dokumentation von Krankheitsschaden vor.

Seit dem Jahr 2001 wird die LuLa-Studie in Zusammenarbeit mit der Lupus Erythematodes Selbsthilfegemeinschaft e.V. (SHG) durchgeführt. Hierbei handelt es sich um eine jährlich durchgeführte patientenzentrierte Befragung einer seit dem Jahr 2005 geschlossenen Kohorte, die Fragen zu multiplen Krankheitsaspekten erhebt. Neben konstanten Fragen u. a. zur Therapie, Krankheitsaktivität („Systemic Lupus Activity Questionnaire“; SLAQ) und HRQoL gibt es jährliche Schwerpunktthemen. Wenn deutschsprachige und validierte Instrumente nicht verfügbar waren, wurden eigene Validierungsprozesse durchgeführt (z. B. „Brief Index of Lupus Damage“, BILD, und SLAQ). Es konnte gezeigt werden, dass die PRO-Angaben der Patienten vergleichbar waren mit den Angaben anderer Kohorten, u. a. auch mit denen der Kerndokumentation des Deutschen Rheumaforschungszentrums. Dies unterstreicht die Qualität und Verwertbarkeit der Patientenangaben bezüglich klinischer Angaben.

In ihrem Beitrag „Selbstmonitoring bei entzündlich-rheumatischen Erkrankungen“ stellen C. Kampling et al. fest, dass bislang in Deutschland wenige Erfahrungen zum Selbstmonitoring existieren. Verschiedene PRO könnten dazu genutzt werden, jedoch herrscht Unklarheit darüber, wie Patienten beim Selbstmonitoring mit gemessenen PRO-Werten umge- 
hen sollen. Empfehlungen nationaler wie internationaler rheumatologischer Fachgesellschaften berücksichtigen das Selbstmonitoring bisher nicht. Für die Rheumatologie stellt sich eine große Evaluations- und Implementierungsaufgabe, damit Patienten durch Selbstmonitoring zunehmend in das Krankheitsmanagement einbezogen werden und somit zu einem besseren Outcome beitragen können.

M. Englbrecht et al. stellen in ihrem Beitrag „Depressivität und Rheuma“ die Frage nach den Möglichkeiten der Messung von Depressivität. Gemessen an den neuesten publizierten hohen Prävalenzraten von Depressionen in Kollektiven von Rheumapatienten ist der potenzielle Nutzen eines entsprechenden Screenings mit Messinstrumenten als hoch einzustufen. Der Artikel befasst sich mit Möglichkeiten und Herausforderungen bei der Erfassung von Depressivität als potenziellem Anhaltspunkt einer depressiven Erkrankung im rheumatologischen Behandlungskontext. Aufbauend auf dem thematischen Hintergrund werden hierzu der gegenwärtige Stand der Wissenschaft sowie eine Auswahl international bekannter Messinstrumente vorgestellt, deren Einsatz auch in der Rheumatologie sinnvoll ist. Ergänzt wird dieser Überblick durch praktische Aspekte, die es insbesondere beim Einsatz von Fragebogen zu beachten gilt, und welche Möglichkeiten für das weitere Vorgehen bei auffälligen Testresultaten bestehen.

Im abschließenden Beitrag von E.Gromnica-Ihle et al. „Wie vermittelt die Deutsche Rheuma-Liga Gesundheitskompetenz?" wird der Weg zu einer patientenzentrierten Gesundheitsversorgung aufgezeigt. Die Patientenorganisation Deutsche Rheuma-Liga sieht die Stärkung der Gesundheitskompetenz der Betroffenen als eine ihrer wichtigsten Aufgaben. Gesundheitskompetente Patienten wollen beim Management ihrer Erkrankung auf der Basis einer partizipativen Entscheidungsfindung gemeinsam mit ihrem Arzt bestimmen, ihre Präferenzen artikulieren und damit auch größere Adhärenz zur gemeinsam festgelegten Behandlungsstrategie zeigen. Betroffene mit Gesundheitskompetenz werden zukünftig sowohl auf der Mikroebene mehr die mündige Kommunikation mit ihren Ärz- ten pflegen als auch auf der Makroebene eine Partizipation bei gesundheitspolitischen Entscheidungen und in der Forschung einfordern.
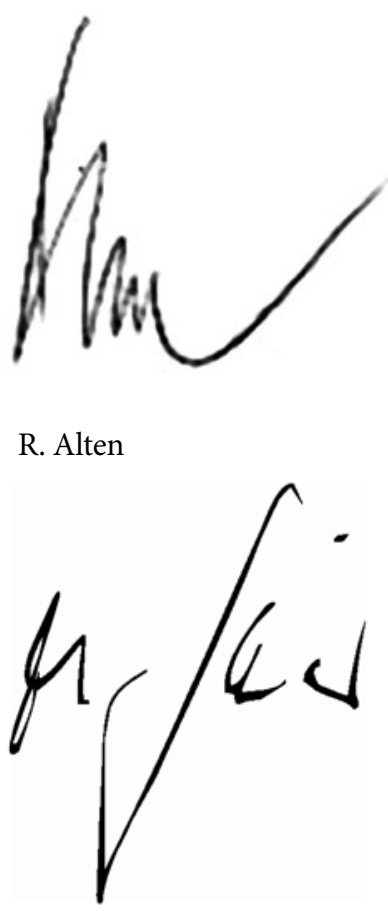

M. Schneider

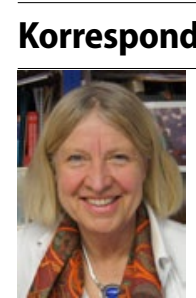

R. Alten

Schlosspark-Klinik, Akademisches

Lehrkrankenhaus der Charité Universitätsmedizin Berlin Heubnerweg 2, 14059 Berlin rieke.alten@ schlosspark-klinik.de

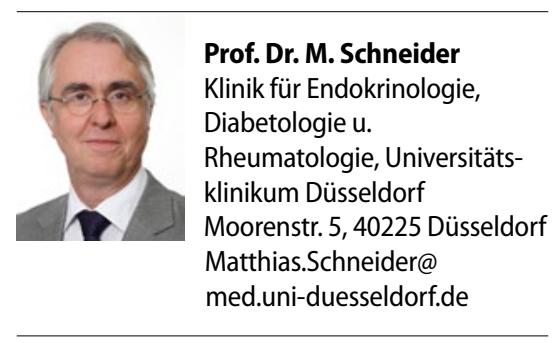

Einhaltung ethischer Richtlinien

Interessenkonflikt. R. Alten und M. Schneider geben an, dass kein Interessenkonflikt besteht.
Renate Hess und Regina Klakow-Franck (Bearb.)

\section{Gebührenordnung für Ärzte} (GOÄ)

Deutscher Ärzte Verlag 2014, 416 S., (ISBN 978-3-7691-3553-4), 39.00 EUR

Das Gebührenverzeichnis zur GOÄ listet Übersichtlich die Bewertungen aller Leistungen in Punktzahlen und die gesamten Euro-Gebühren, wie den Einfachsatz, die Steigerungssätze sowie die Gebühren für Standart- und Basistarif der Privaten Krankenversicherungen auf. Außerdem ist das Verzeichnis der Analogen Bewertungen der Bundesärztekammer erforderlich für die Abrechnung moderner Untersuchungsund Behandlungsmethoden. Zusätzlich finden Sie den aktuellen Sachstand zur GOÄ-Reform übersichtlich in der Erläuterung der neuen Rahmenvereinbarung zwischen Bundesärztekammer und PKVVerband. Zum ersten Mal gibt es in dieser Auflage einen IGeL-Ratgeber von Bundesärztekammer und Kassenärztlicher Bundesvereinigung als Orientierungshilfe für Ärzte und Patienten. Somit ist diese Ausgabe des Deutschen Ärzte-Verlages eine gute Grundlage für die Privatliquidation und die IGeL-Leistungen.

D. A. Groneberg (Frankfurt) 